\title{
Effect of particle surface friction on nonlocal constitutive behavior of flowing granular media
}

\author{
Ken Kamrin · Georg Koval
}

Received: 13 January 2014 / Revised: 1 April 2014 / Accepted: 11 April 2014 / Published online: 2 May 2014

(C) Springer International Publishing Switzerland 2014

\begin{abstract}
A recently proposed nonlocal rheology for dense granular flow, based on the concept of nonlocal granular fluidity, has demonstrated predictive capabilities in multiple geometries. This work is concerned with determining how the parameters of this continuum model arise from the properties of the grains themselves. We perform a controlled study investigating how the surface friction of the grains influences the continuum parameters, with a focus on how the nonlocal amplitude, the model's one new parameter, is affected. This is achieved comparing two-dimensional discrete-element simulations of flowing disks to numerical solutions of the model in planar shear and several annular shear geometries. A multistep calibration scheme for the continuum parameter extraction is developed and implemented. Results indicate the nonlocal amplitude varies less than an order of magnitude over a wide range of surface frictions, with a slight tendency to increase as surface friction decreases, particularly in a regime of small surface friction. Our data also show that the stress and flow-rate variables deviate little from a local relationship as surface friction vanishes, which corroborates certain existing experimental findings.
\end{abstract}

Keywords Granular media $\cdot$ Rheology $\cdot$ Nonlocal

\footnotetext{
K. Kamrin ( $\square)$

Department of Mechanical Engineering, MIT, Cambridge, MA 02139, USA

e-mail: kkamrin@mit.edu

G. Koval

ICube Laboratory, National Institute of Applied Sciences, Strasbourg, France
}

\section{Introduction}

Dry granular materials are ubiquitous in day-to-day life (geotechnical matter, raw materials, food grains, pharmaceuticals) and are second only to water as the world's most handled industrial material. While modeling efforts for these materials go back over two centuries, originating with Coulomb's pioneering work, a precise continuum description for granular deformation has remained an elusive goal. In recent years, however, notable progress has been made on the specific question of how to predict well-developed flows of dense, dry granular media. A major step was the advent of the "inertial rheology," a local constitutive description of flowing grains, ascribing a direct relationship between the local stress state and the strain-rate $[7,13]$. The particular relationship that emerges can be understood through dimensional analysis of planar simple shearing of a material composed of stiff, round grains with frictional viscoelastic contacts, and takes the form of a dimensionless relationship

$\mu=\mu_{l o c}(I), \quad I=\dot{\gamma} \sqrt{m / P}, \quad \mu=\tau / P$,

where $\mu$ is the ratio of shear stress $\tau$ and normal pressure $P, I$ is the inertial number, $\dot{\gamma}$ is the shear rate, and $m$ is the mean mass of a grain. This relation agrees with earlier scaling relations stemming back to Bagnold [4]. The inertial number can be understood as a ratio of the macroscopic time-scale of applied shearing, to the microscopic or inertial time. The precise function for $\mu_{l o c}(I)$ is empirically fit from planar simple shearing data and is characterized by a yield condition $\mu_{s}$ such that $\mu_{\text {loc }}(I \rightarrow 0)=\mu_{s}$, rendering the relation akin to that of a pressure-dependent yield stress fluid. This rheology has also been coupled to a granular elasticity relation [14], thereby closing the system mathematically to compute stress and velocity in flowing or static grains, producing a general mechanical law. 
The inertial rheology works well in describing uniform flows (e.g. planar shearing) over a wide range of flow rates [7]. It can also be isotropically extended to a fully $3 \mathrm{D}$ rheology and is able to represent nonuniform flows at rapid rates [13]. However, in slower, quasi-static zones of flow (roughly $I<10^{-3}$ ) in nonuniform flow geometries, the one-to-one inertial relation between $\mu$ and $I$ is violated [16], and inertial rheology flow predictions are incorrect. The behavior displayed in these zones is unexplainable through any local relation because the bulk stress/strain-rate behavior appears to vary with the macroscopic geometry, even when the local kinematics are identical. This has motivated the search for an appropriate nonlocal model, able to account for such geometric effects, by inclusion of an explicit length-scale based on the mean grain diameter, $d$.

A nonlocal rheology that has recently emerged, the "nonlocal granular fluidity" (NGF) model, appears to have addressed this issue, and demonstrates the ability to quantitatively predict granular flows in many disparate geometries, in both rapid and quasi-static regimes [10,15]. Its predictions have been verified in $2 \mathrm{D}$ and $3 \mathrm{D}$ as compared to both discrete-particle simulations [15] and experiments [10]. Of note, it is the first continuum model to quantitatively describe all known flow data in the complex split-bottom family of geometries [10]. The NGF model's form takes inspiration from the form of other fluidity models for emulsions, derived from statistical physics arguments [5] rooted in soft glassy rheology principles [23].

Specifically, NGF (in its reduced scalar form) is composed of the system

$\dot{\gamma}=g \mu, \quad g_{l o c}(\mu, P)=\frac{\dot{\gamma}_{l o c}(\mu, P)}{\mu}=\frac{\mu_{l o c}^{-1}(\mu) \sqrt{P / m}}{\mu}$,

$g=g_{l o c}+\xi^{2} \nabla^{2} g$.

The field $g$ is the granularfluidity, and $\xi$ is the plastic cooperativity length, which is proportional to $d$. Note that in planar shear, flow gradients vanish and the above reduces appropriately to the local rheology, but in the presence of gradients, the Laplacian term "spreads" fluidity based on $\xi$. As verified in multiple ways in $[10,15], \xi$ is in fact not a constant length; as in [10] we use a form inspired by [5], i.e.

$\xi=\frac{A}{\sqrt{\left|\mu-\mu_{s}\right|}} d$,

a form consistent with past work on jamming in amorphous media $[8,17,18,21,24]$ in that it diverges approaching a yield, or jamming point. The parameter $A$, the dimensionless nonlocal amplitude, is the only new parameter in the model, which quantifies the cooperativity in the flow.

With the successes of NGF, a key question to ask is: What is the connection between properties of the grains themselves and the continuum parameters/functions of the model, most importantly the new parameter $A$ ? To be able to answer this question is potentially of great value, as it would provide more complete upscaling between the microand macro-scales, and moves us closer to the ability to predict a granular flow upon mere examination of the individual grains. There are limitless grain properties to consider; we are aware of past studies that have looked at connections to the grain shape [2,3], polydispersity [22], and surface roughness [6], but all have focused on the local continuum response only. In this paper, we seek to provide a first step to the broader, nonlocal, scenario by investigating how the NGF model parameters depend on a single pertinent grain parameter while controlling all others. The property we will vary is the surface friction coefficient, $\mu_{\text {surf }}$, which is a property expected to have a nontrivial effect down into the quasistatic regime for which the effect of nonlocality is the most obvious.

To extract the continuum parameters, our study will utilize discrete-element method (DEM) simulations of a material composed of quasi-monodiserse 2D disks. Our general strategy is to simulate flows in planar shear to measure the local response function $\mu_{l o c}(I)$, and then to fit $A$ by simulating the same material in a family of annular shear geometries, which brings out the nonlocal phenomena clearly. To fit $A$ we must calculate NGF solutions for comparison against the DEM, which we do numerically using Mathematica 10. Due to the symmetry of the annular cell geometry, the fluidity partial differential equation reduces to an ordinary differential equation, greatly simplifying the numerical solution process.

Details of the discrete-particle simulations are described next, followed by details of the the parameter extraction and results.

\section{Discrete-element simulations}

\subsection{Studied geometries}

The model is compared to two-dimensional annular and planar systems (see Fig. 1). The annular geometry is defined by its inner radius $R$ and outer radius $R o$. The gap between the walls is equal to $50 d$ on the planar geometry and equal to $2 R$ on the annular geometry $(R o=3 R)$, which avoids any disturbing effect of the walls on the material behavior. The granular material is composed of dissipative disks of average diameter $d$ (a polydispersity of $20 \%$ prevents crystallization) and average mass $m$. The shear stress is fully mobilized by the relative motion of two rough walls made of contiguous glued grains of a diameter equal to $2 d$. We prescribe the tangential inner wall velocity $V_{w}$ and the normal stress $P_{w}$ (indirectly through the motion of the outer wall). The value of $R o$ slightly fluctuates with a velocity $\dot{R} o=\left(P(R o)-P_{w}\right) / g_{p}$, 


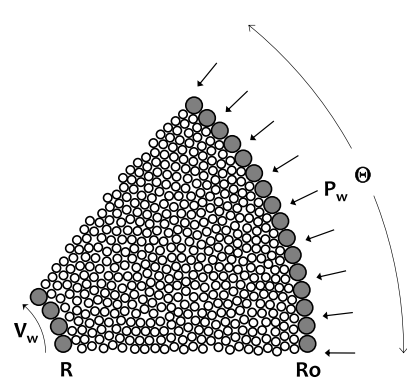

(a)

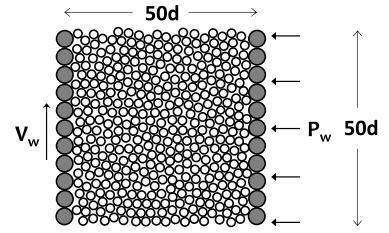

(b)

Fig. 1 Two-dimensional a annular and $\mathbf{b}$ planar shear cells

Table 1 Dimensions of the annular shear cells

\begin{tabular}{lrl}
\hline & $R_{i} / d$ & $\Theta /(2 \pi)$ \\
\hline$R_{25}$ & 25 & $1 / 4$ \\
$R_{50}$ & 50 & $1 / 8$ \\
$R_{100}$ & 100 & $1 / 12$ \\
\hline
\end{tabular}

where $P(R o)$ is the normal stress at the vicinity of the outer wall and $g_{p}$ is a viscous damping parameter. We define the (measured) shear stress at the inner wall $S$.

We take advantage of the symmetry of the simulated systems introducing periodic boundary conditions along the tangential direction. The annular cell is represented by an angular sector $\Theta$, while the plane shear cell presents a width equal to $50 d$ (see [16] for more details).

Table 1 describes the dimensions of the annular shear cells.

\subsection{System parameters}

The contacts are described by a standard spring-dashpot law defined by the damping coefficient $g_{n}$, the coefficient of friction $\mu_{\text {surf }}$, and the elastic stiffness parameters $k_{n}$ and $k_{t}$ (see Fig. 2). Physically we can relate $g_{n}$ to the coefficient of restitution $e\left(g_{n}=-2 \ln e \sqrt{m k_{n} /\left(\pi^{2}+\ln ^{2} e\right)}\right)$ which

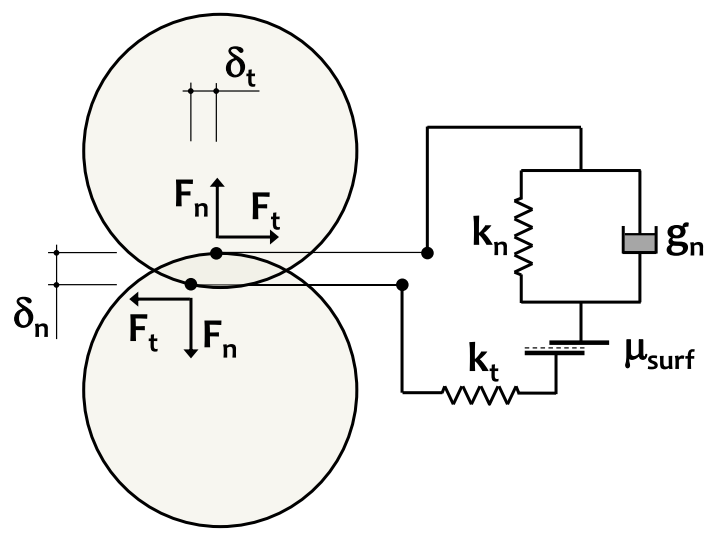

Fig. 2 Contact parameters describes the dissipation of energy during a shock (with duration $\left.\tau_{c}=\pi / \sqrt{2 k_{n} / m-\left(g_{n} / m\right)^{2}}\right)$.

The components of the contact forces $\left(F_{n}\right.$ and $\left.F_{t}\right)$ depend on normal and tangential components of the contact displacements (respectively $\delta_{n}$ and $\delta_{t}$ ) and also on the time derivative of the normal displacement $\dot{\delta}_{n}$. For two particles in contact $\left(\delta_{n} \geq 0\right)$, the normal component $F_{n}=k_{n} \delta_{n}+g_{n} \dot{\delta}_{n}$ is always repulsive $\left(F_{n} \geq 0\right)$, while the tangential component $F_{t}=k_{t} \delta_{t}$ is limited by Coulomb friction $\left(-k_{n} \delta_{n} \mu_{\text {surf }} \leq\right.$ $\left.F_{t} \leq k_{n} \delta_{n} \mu_{\text {surf }}\right)$. The motion of the particles is discretized using Gear's order three predictor-corrector algorithm [1]. A time step equal to $\tau_{c} / 100$ was chosen to perform simulations with standard molecular dynamics method as in Refs. $[7,15,16]$.

Dimensional analysis allows us to reduce the number of parameters to a few dimensionless quantities. We adopt the relation $k_{t} / k_{n}=0.5$ and $e=0.1$, two parameters with nearly no influence in dense granular flows [7]. We set the relation between the normal stiffness and the confining pressure $k_{n} / P_{w}=10^{4}$ in order to simulate quasi-rigid grains. The motion of the outer wall is controlled by the dimensionless number $g_{p} / \sqrt{m k_{n}}=0.1$ which guarantees a permanent contact between the wall and the material avoiding any eventual disturbance of the granular behavior.

We analyze the effect of different materials with a wide range of coefficients of friction $\mu_{\text {surf }}(0,0.025,0.05,0.1$, $0.2,0.4$ and 0.8 ) in all shear cells.

Similar to the inertial number $I$, we can define the dimensionless wall velocity $V$ :

$V=\frac{V_{w}}{d} \sqrt{\frac{m}{P_{w}}}$

which describes the shear state in the scale of the system.

The local behavior of each granular material $\mu_{l o c}(I)$ is obtained by plane shear simulations at different values of $V$ $(0.005,0.025,0.05,0.25,0.5,2.5,5.0$ and 7.5$)$. The nonlocal behavior is obtained in the annular cells with $V=2.5$, where a transition between inertial to quasi-static regimes is observed from the inner to the outer wall.

\subsection{Steady shear states}

Initially, the particles are randomly disposed inside the cells, producing very loose samples. As observed in [16], the system reaches a steady state, characterized by constant time averaged profiles of velocity and stress, after a short transient inferior to the shear length $V \Delta t \approx 50$ (where $\Delta t$ is the simulation time). In practice, we adopt the conservative steady state condition $V \Delta t=100$. All quantities result from a time average of 400 steps distributed over the distance $V \Delta t \approx 200$. The material bulk behavior is obtained from space averages of the whole sample (excepting the very first 
layers near the walls) in plane shear. Annular results are analyzed by radial profiles smoothed through central moving averages of $d$ length (see Appendix for more details).

\section{Continuum parameter extraction}

Because the nonlocal effect is characterized by its ability to remove uniqueness of the local rhelogical connection between $\mu$ and $I$ apparent in gradient-free flow geometries, it is sensible to calibrate $A$ based on how the $\mu$ and $I$ fields in the annular cell differ from the $\mu_{l o c}$ relation in planar shear. Our calibration strategy takes a few steps. For each value of $\mu_{\text {surf }}$,

1) The inertial rheology, given by the function $\mu_{l o c}$, is fit by conducting a sequence of planar shear DEM simulations of different prescribed $I$, measuring $\mu$. The result is then fit to a general mathematical form; we have found the following form appropriate,

$\mu=\mu_{l o c}(I)=\mu_{0}+b I+\left(\mu_{s}-\mu_{0}\right) \exp (-I / C)$,

see Fig. 3 for example. A simple linear relation between $\mu$ and $I$ is often assumed, but the above nonlinear form permits us to better fit data for low $\mu_{\text {surf }}$ grains for which past studies have revealed a non-negligible curvature of the $\mu_{l o c}$ function at low $I$ [7]. We note that curvature of the $\mu$ versus $I$ relation is also observed for large $I$ [12], but in a range outside that of our current interest.

2) Three separate annular shear flow DEM simulations are conducted, for $R=25,50$, and $100 \mathrm{~d}$. Coarse-grained data is recorded when steady state is reached. The stress distributions obtained, for each $R$, are fit to the mechanically justified relations

$$
\mu(r)=\frac{S_{w} R^{2}}{r^{2} P(r)}, \quad P(r) \cong P_{w} .
$$

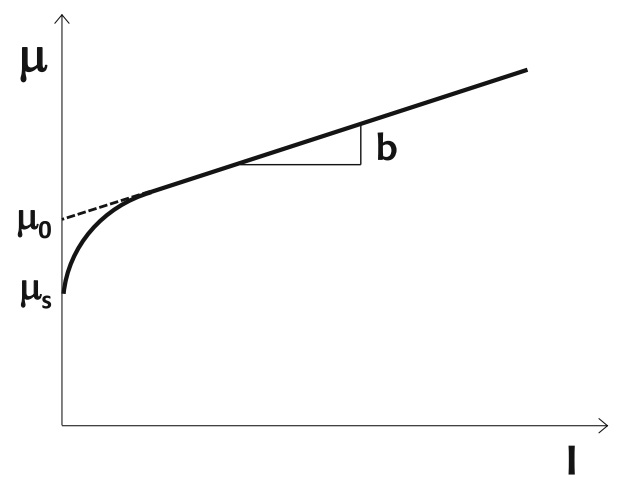

Fig. 3 Relationship between local parameters and the fit-form for the inertial rheology
The former relation is implied from torque balance, and always well-fit to DEM data, and the latter is valid based on our own data as well as others' in this particular geometry $[15,16]$. Here, $S_{w}$ is the shear stress acting on the inner wall.

3) Numerical simulation of NGF is begun by inputting the above fit for $\mu(r)$, the local flow-rate $\dot{\gamma}_{l o c}(r)$ as obtained applying the inertial rheology, and a guess for $A$. The fluidity system, Eq. 2, is then solved for $g(r)$, in the three annular shear geometries, assuming $g=g_{l o c}$ on the boundaries. We have used this boundary condition in past annular shear flow simulations with success [15], however, the question of the precise form of the fluidity boundary condition is still open. One other condition has been used before, the Neumann condition $\partial g / \partial n=0$ [10]. Both are simple and pass the minimal test of yielding a uniform $g$ field in planar shear, as one would expect for 'ideal' walls with small wall effects, and result in very similar solutions overall, with small differences only within a few grain diameters of a boundary. The latter point is owed to the nature of the fluidity PDE being of source-diffusion type, such that boundary effects tend to be dominated over a short distance by the bulk source. Once $g(r)$ is computed, $I(r)$ is obtained trivially from its definition in Eq. 1.

4) Plots of $\mu$ versus $I$ are made for all three annular shear NGF solutions, and compared directly against scatterplots of those of the DEM. $A$ is varied under a simple bisection calibration approach until the NGF solutions best match those of the DEM. We judge the match over two decades of $I$, from 0.0005 to 0.05 , which is low enough to cover a broad range of behavior dominated by the nonlocal effect but still large enough to capture the transition zone where the local influence is diminishing.

\section{Results}

Our tests cover seven different surface roughness values: $\mu_{\text {surf }}=0,0.025,0.05,0.1,0.2,0.4,0.8$. Figure 4 shows the results of our fitting of the inertial rheology to the planar shear DEM data for each $\mu_{\text {surf }}$. The parameters corresponding to each fit can be viewed in Table 2 (Local). Overall, the local fitting form appears sufficient. In some of the DEM data a smalll oscillation in the $\mu$ versus $I$ behavior is apparent for very low $I$. This behavior would be in line with certain experimental results [9], though most existing DEM data in this geometry display monotonicity down to low $I$ [19]. At this point we would rather not speculate on whether this is a physical or numerical perturbation. Figure 6 indicates the clear trend of the bulk static friction coefficient, $\mu_{s}$, monotonically decreasing albeit nonlinearly with $\mu_{\text {surf }}$, in good agreement with past work on roughness effects in the 


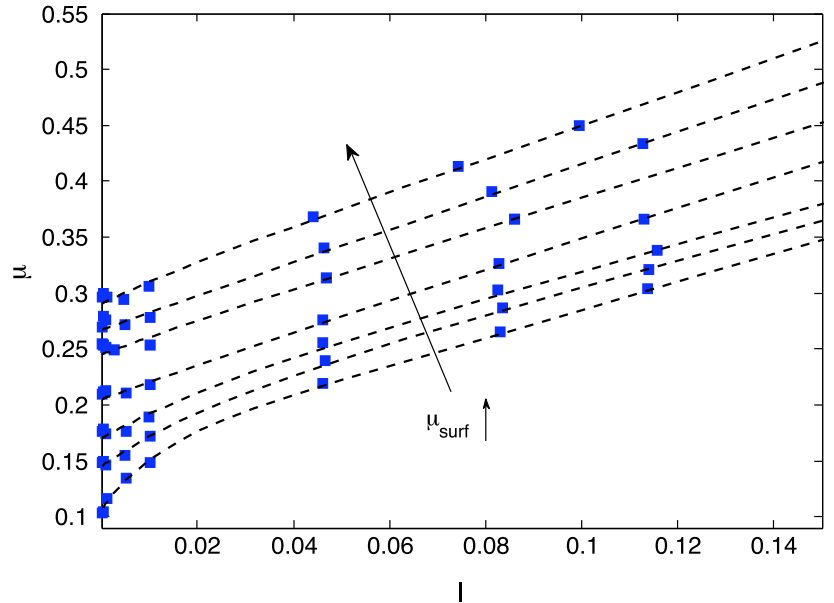

(a)

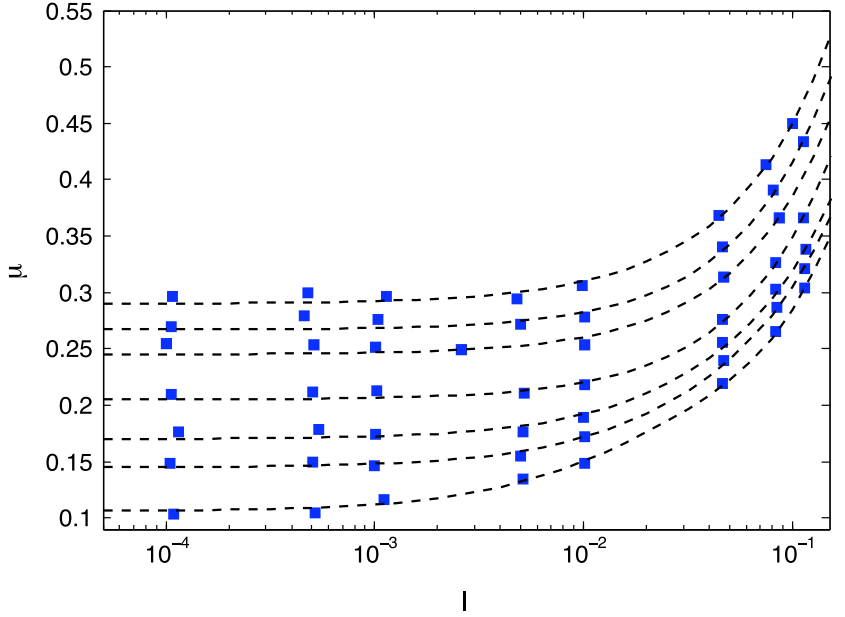

(b)

Fig. 4 Planar shear flows: DEM data (squares) and selected fit functions per Eq. 5 (dashed lines) corresponding to all seven particle surface frictions, displayed simultaneously in a linear and $\mathbf{b}$ semilog

Table 2 Calibrated model parameters for all different grain frictions

\begin{tabular}{llllll}
\hline$\mu_{\text {surf }}$ & \multicolumn{4}{l}{ Continuum parameters } \\
\cline { 2 - 6 } & Nonlocal & Local & & & \\
& $A$ & $\mu_{s}$ & $\mu_{0}$ & $b$ & $C$ \\
\hline 0.8 & 0.84 & 0.290 & 0.300 & 1.50 & 0.014 \\
0.4 & 0.80 & 0.265 & 0.270 & 1.45 & 0.025 \\
0.2 & 0.85 & 0.245 & 0.250 & 1.35 & 0.020 \\
0.1 & 0.83 & 0.205 & 0.215 & 1.35 & 0.033 \\
0.05 & 0.97 & 0.170 & 0.200 & 1.20 & 0.025 \\
0.025 & 1.03 & 0.145 & 0.185 & 1.20 & 0.022 \\
0 & 1.05 & 0.106 & 0.160 & 1.25 & 0.011 \\
\hline
\end{tabular}

inertial rheology [6]. The decreasing amount of curvature in the plots for larger $\mu_{\text {surf }}$ is captured by the decreasing separation between $\mu_{s}$ and $\mu_{0}$ as $\mu_{\text {surf }}$ increases.

The calibrated values of the nonlocal amplitude, $A$, are also presented in Table 2 along with the corroborating plots in Fig. 5, where NGF outcomes for $\mu$ versus $I$ in the four different geometries are compared directly to those of the DEM, for each $\mu_{\text {surf }}$. The degree of agreement between DEM data and the NGF model, for each $\mu_{\text {surf }}$, as seen in the figure, lends confidence to the fits for $A$ and the effectiveness of the NGF model. However, there is some bumpiness evident in the extracted $A$ versus $\mu_{\text {surf }}$ relation (Fig. 6), which could arise from sampling uncertainties in the DEM combined with the sensitive four-step calibration process used to obtain $A$. The latter point reflects the nature of $A$ within the system as that of a coefficient of a high-order derivative of an implicitly solved variable $g$, which precludes a straightforward measurement scheme for $A$ from DEM data.

In Fig. 5, it is interesting to note that the degree of deviation from the local (i.e. planar shear) data appears to decrease as $\mu_{\text {surf }}$ decreases, yet $A$ is actually increasing with decreasing $\mu_{\text {surf }}$ (see Fig. 6). This may seem counterintuitive, but it can be explained. Indeed, if all all other model parameters are held fixed, the extent of deviation from locality always grows with $A$ and vanishes as $A$ vanishes, in a given geometry. However, as $\mu_{\text {surf }}$ varies, the other NGF model parameters are not staying fixed. In other words, if deviation from locality were assigned a measure, one might say $A$ acts as an amplifier of deviation, but the term it amplifies depends on the other model parameters.

Our results suggest three conclusions about $A$. The first is that, unless the surface friction is very small $(<0.1)$, the nonlocal amplitude does not appear to vary to a significant extent with $\mu_{\text {surf }}$ (neither does $\mu_{s}$ ). Secondly, in the case of small $\mu_{\text {surf }}, A$ actually displays an increasing behavior as $\mu_{\text {surf }}$ decreases, as previously noted. The third is that the overall size of $A$ remains order-one over the range of $\mu_{\text {surf }}$ considered-even as the increasing behavior of $A$ with decreasing $\mu_{\text {surf }}$ becomes evident, this increase is much less than an order of magnitude. The order-one nature of $A$ agrees with past NGF work $[10,15]$.

The various curves relating $\mu$ and $I$ displayed in Fig. 5 may shed insight on observations that have been made in flows of suspensions. As discussed in [11,20], dense suspension flows tend to obey a single local rheology rather well, even in small geometries (relative to the particle size). This notion lies in contrast to our observations with frictional dry granular media for the majority of $\mu_{\text {surf }}$ cases. In view of Fig. 5, as surface friction drops, we observe that the rheological curves of granular matter in different geometries, i.e. the correspondences between $\mu$ and $I$, begin to collapse together. In fact, in the $\mu_{\text {surf }}=0$ case, the dispersion between the curves seems to vanish altogether and a dominantly local response is evident. Hence, the dispersion of $\mu$ versus $I$ loci over different 

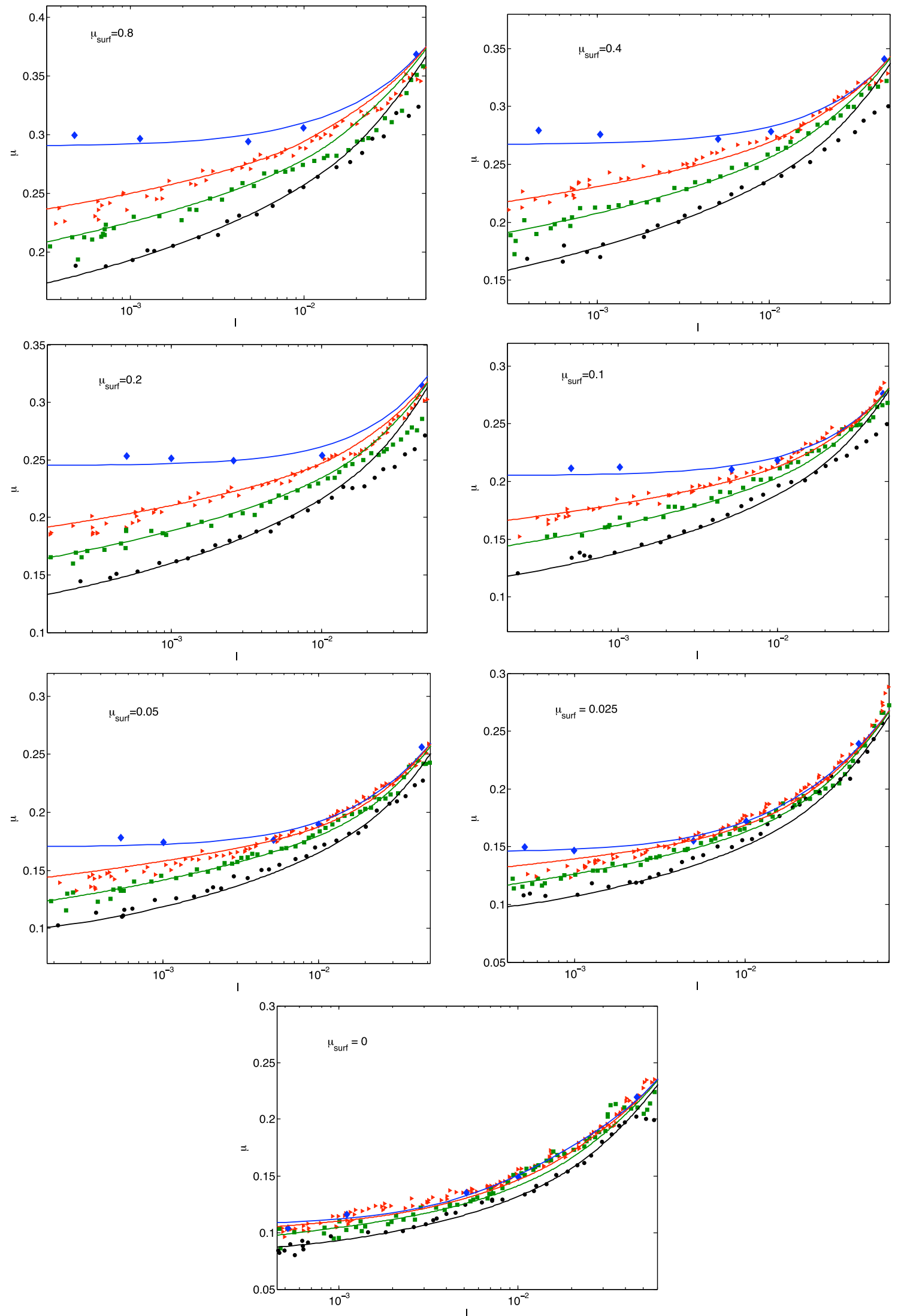

Fig. 5 Calibrated NGF model [c.f. Table 2] (lines), versus DEM data (symbols) for planar shear (diamond) and annular shear geometries with $R=25 d$ (circle), 50d (square), 100d (right pointing triangle). Corresponding geometries are color-matched 


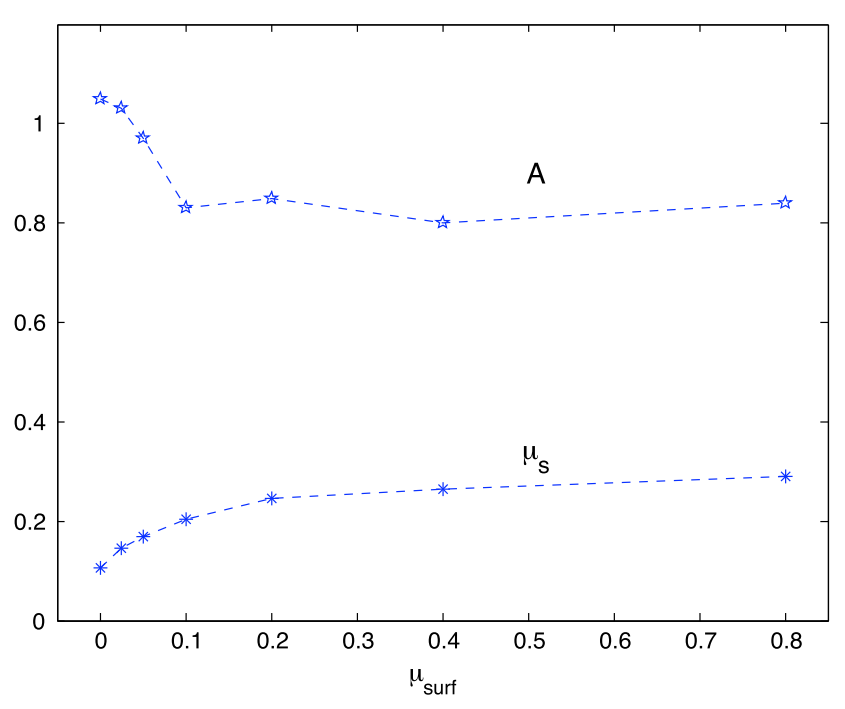

Fig. 6 Dependence of the nonlocal amplitude and the static planar shear strength on surface friction

geometries diminishes in circumstances where particle interactions lack a tangential force component. In suspensions, the suspending fluid may behave as a lubrication layer between adjacent particles, which reduces tangential drag. It therefore stands to reason that the lack of geometry-dependence among the rheological curves in a dense suspension may be due to the same effect of reduced surface drag we observe herein.

\section{Summary and future work}

We have performed the first upscaling study to link the recent NGF model for granular flow directly to particle properties. Our parameter study has focused on the effect of particle roughness, corroborating past work on local rheology dependences and revealing a number of conclusions about its effect on the nonlocal amplitude. Perhaps the over-riding point is that $A$ is influenced by $\mu_{\text {surf }}$ but not tremendously. This result would support the idea that $A$ is mostly a reflection of the geometric constraints involved in particles moving past other particles in a dense packing, and not so much a product of the contact interaction details. Although $A$ slightly increases as $\mu_{\text {surf }}$ decreases, Fig. 5 indicates that the rheological curves obtained from different geometries are in fact collapsing more and more as $\mu_{\text {surf }}$ decreases. As we have noted, this observation corroborates the high degree of collapse observed in some suspension flows, wherein liquid lubrication between grains reduces tangential contact force.

It remains future work to continue such a study to analyze the effects of varying other particle properties individually, or combinations of properties. Obvious properties to consider next would be particle shape and polydispersity, though we

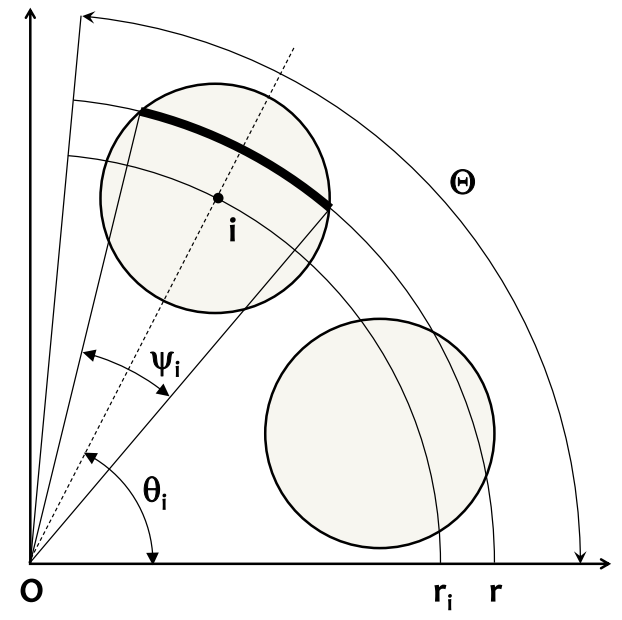

Fig. 7 Weight function $\psi_{i}(r)$

note that size-segregation effects will complicate a polydispersity study under our current calibration approach, which involves imposition of a nonuniform flow field, which can induce spatial variations in the size distribution. It would also be interesting to expand this analysis to three dimensions, where past results suggest the nonlocal amplitude will be overall smaller [10,15]. From a physical standpoint, it also remains to attempt a theoretical understanding for these results. This is a non-trivial task, but might be achievable by revisiting how particle contact interactions factor in to the existing theoretical derivation for the nonlocal fluidity model.

\section{Appendix: Averaging method}

The radial profiles of different quantities are obtained by an averaging procedure over coordinate $\theta$ along the coordinate $r$, where each of the $n$ grains $i$ is taken into account (see Fig. 7). We define a weight function $\psi_{i}$ as the intercepted angle defined on Fig. 7, where $\cos \left(\psi_{i}(r) / 2\right)=\left(r^{2}+r_{i}^{2}-\right.$ $\left.d_{i}^{2} / 4\right) /\left(2 r_{i} r\right)$ for a disk of diameter $d_{i}$ and a radial position $r_{i}$.

We consider the polar basis $\underline{e}_{r}(\phi)=(\cos \phi, \sin \phi)$ and $\underline{e}_{\theta}(\phi)=(-\sin \phi, \cos \phi)$. Hence, the radial profile of the orthoradial velocity is

$v_{\theta}(r)=\frac{1}{\sum_{i=1}^{n} \psi_{i}(r)} \sum_{i=1}^{n} \int_{\theta_{i}-\psi_{i} / 2}^{\theta_{i}+\psi_{i} / 2} \underline{v}_{i} \cdot \underline{e}_{\theta}(\phi) d \phi$.

The stress tensor of each grain $i$ is defined as

$\underline{\underline{\sigma}}^{i}=\frac{1}{A^{i}} \sum_{j \neq i} \underline{F}_{i j} \otimes \underline{r}_{i j}$ 
with the particle area $A^{i}=\pi d_{i}^{2} / 4$, the contact force (between two particles $i$ and $j$ ) $\underline{F}_{i j}$ and the corresponding position and $\underline{r}_{i j}$. The radial profiles of the components of the stress tensor are

$\sigma_{\alpha \beta}(r)=\frac{1}{\Theta} \sum_{i=1}^{n} \int_{\theta_{i}-\psi_{i} / 2}^{\theta_{i}+\psi_{i} / 2} \underline{e}_{\alpha}(\phi) \underline{\sigma}^{i} \underline{e}_{\beta}(\phi) d \phi$.

\section{References}

1. Allen MP, Tildesley DJ (1987) Computer simulation of liquids. Oxford University Press, Oxford

2. Azéma E, Radjai F, Saussine G (2009) Quasistatic rheology, force transmission and fabric properties of a packing of irregular polyhedral particles. Mech Mater 41(6):729-741

3. Azéma E, Estrada N, Radjai F (2012) Nonlinear effects of particle shape angularity in sheared granular media. Phys Rev E 86(4):041301

4. Bagnold RA (1954) Experiments on a gravity free dispersion of large solid spheres in a newtonian fluid under shear. Proc R Soc Lond Ser A 225:49-63

5. Bocquet L, Colin A, Ajdari A (2009) Kinetic theory of plastic flow in soft glassy materials. Phys Rev Lett 103:036001

6. da Cruz F (2004) Friction and jamming in granular flows. PhD thesis, Ecole Nationale des Ponts et Chaussees, Marne a la vallée, France

7. da Cruz F, Emam S, Prochnow M, Roux J, Chevoir F (2005) Rheophysics of dense granular materials: discrete simulation of plane shear flows. Phys Rev E 72:021309

8. Dahmen KA, Ben-Zion Y, Uhl JT (2011) A simple analytic theory for the statistics of avalanches in sheared granular materials. Nat Phys 7:554-557

9. Dijksman JA, Wortel GH, van Dellen LTH, Dauchot O, van Hecke M (2011) Jamming, yielding, and rheology of weakly vibrated granular media. Phys Rev Lett 107(10):108303
10. Henann D, Kamrin K (2013) A predictive, size-dependent continuum model for dense granular flows. PNAS 110(17):6730-6735

11. Huang Nicolas, Bonn Daniel (2007) Viscosity of a dense suspension in couette flow. J Fluid Mech 590:497-507

12. Jop P, Forterre Y, Pouliquen O (2005) Crucial role of side walls for granular surface flows: consequences for the rheology. J Fluid Mech 541:21-50

13. Jop P, Forterre Y, Pouliquen O (2006) A constitutive law for dense granular flows. Nature 441:727

14. Kamrin K (2010) Nonlinear elasto-plastic model for dense granular flow. Int J Plast 26:167-188

15. Kamrin K, Koval G (2012) Nonlocal constitutive relation for steady granular flow. Phys Rev Lett 108:178301

16. Koval G, Roux J-N, Corfdir A, Chevoir F (2009) Annular shear of cohesionless granular materials: from the inertial to quasistatic regime. Phys Rev E 79:021306

17. Lemaitre A, Caroli C (2009) Rate-dependent avalanche size in athermally sheared amorphous solids. Phys Rev Lett 103:065501

18. Lois G, Carlson JM (2007) Force networks and the dynamic approach to jamming in sheared granular media. Europhys Lett 80:58001

19. Midi GDR (2004) On dense granular flows. Eur Phys J E 14:341365

20. Nordstrom KN, Verneuil E, Arratia PE, Basu A, Zhang Z, Yodh AG, Gollub JP, Durian D (2010) Microfluidic rheology of soft colloids above and below jamming. Phys Rev Lett 105(17):175701

21. Picard G, Ajdari A, Lequeux F, Bocquet L (2005) Slow flows of yield stress fluids: complex spatiotemporal behavior within a simple elastoplastic model. Phys Rev E 71:010501

22. Rognon PG, Roux J-N, Naaïm M, Chevoir F (2007) Dense flows of bidisperse assemblies of disks down an inclined plane. Phys Fluids 19:058101

23. Sollich P, Lequeux F, Hébraud P, Cates M (1997) Rheology of soft glassy materials. Phys Rev Lett 78:2020-2023

24. Staron L, Vilotte J-P, Radjai F (2002) Preavalanche instabilities in a granular pile. Phys Rev Lett 89(20):204302 